\title{
Delay Colouring in Quartic Graphs
}

\author{
Katherine Edwards* \\ katherine.edwards2@gmail.com
}

\author{
W. Sean Kennedy \\ william.kennedy@nokia-bell-labs.com
}

Submitted: Oct 2, 2018; Accepted: Apr 16, 2019; Published: Aug 7, 2020

(C) The authors. Released under the CC BY-ND license (International 4.0).

\begin{abstract}
Haxell, Wilfong, and Winkler conjectured that every bipartite graph with maximum degree $\Delta$ is $(\Delta+1)$-delay-colourable. We prove this conjecture in the special case $\Delta=4$.
\end{abstract}

Mathematics Subject Classifications: 05C15

\section{Introduction}

The TWIN network architecture (see Section 2 for a brief overview) was introduced by Saniee and Widjaja [9] to maximize bandwidth and simplify network complexity in backbone optical transmission networks. It relies on an efficient scheduler that ensures transmitting nodes send optical bursts to at most one destination at a time and receive at most one optical burst at a time while taking into account the differing transmission times, or delays, between source-destination pairs in the network. Based on this, Haxell, Wilfong, and Winkler [6] introduced the notion of delay colouring as follows. Suppose we are given a bipartite (multi)graph $G=(V=(X, Y), E)$ and a delay function $r: E(G) \rightarrow \mathbb{N}$. We say that $(G, r)$ is $\tau$-delay-colourable if there is a function $f$ from $E$ to the integers modulo $\tau$ such that for each vertex $x \in X$, the elements of the collection $\left\{f_{e}: e \in E, x \in e\right\}$ are distinct; and for each vertex $y \in Y$, the elements of the collection $\left\{f_{e}+r_{e}(\bmod \tau): e \in E, y \in e\right\}$ are distinct. The delay chromatic number $\chi_{d}(G)$ is the minimum $\tau$ such that $(G, r)$ is $\tau$-delay-colourable for each delay function $r$.

Clearly, $\chi_{d}(G) \geqslant \chi^{\prime}(G) \geqslant \Delta(G)$, where $\chi^{\prime}$ and $\Delta$ respectively represent the chromatic index and maximum degree. It is well known that $\chi^{\prime}(G)=\Delta(G)$. Haxell et al. point out that in general $\chi_{d}$ can exceed $\Delta$, since in a $\Delta$-regular graph, the (multi)set of values $\{f(e): e \in E\}$ would have to be identical to the set of values $\left\{f(e)+r_{e}: e \in E\right\}$, and this can only be achieved if $\sum_{e \in E} r_{e}=0(\bmod \Delta)$; it's easy to construct examples of $r$ where this condition doesn't hold (see Fig. 1 for an example). They offered the following conjecture.

\footnotetext{
${ }^{*}$ Nokia Bell Labs, Murray Hill, NJ, USA
} 


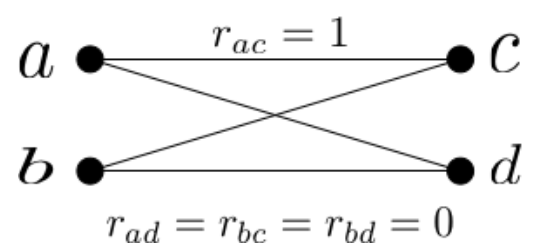

Figure 1: An example graph $G$ satisfying $\chi_{d}(G)>\Delta(G)$.

Conjecture 1. Let $G=(V=(X, Y), E)$ be a bipartite (multi)graph of maximum degree $\Delta$. Then $\chi_{d}(G) \leqslant \Delta+1$.

In the case where $|X|=|Y|=1$, the conjecture can be shown using a theorem of Marshall Hall [5]. Hall shows that given an Abelian group of order $m$ and a multiset of m elements $\left\{r_{e}\right\}$ that sum to identity, there is a permutation $\pi$ of the group for which $\pi_{e}+r_{e}$ are all distinct. In this special case the conjecture follows by adding an additional edge whose delay is such that the delays sum to 0 and applying Hall's result. Conjecture 1 is easy to check for graphs of maximum degree at most 2, and Georgakopoulos proved it for graphs with $\Delta=3[3]$.

Here, using a different approach, we prove it in the case $\Delta=4$.

Theorem 2. Suppose $G$ has maximum degree 4. Then $\chi_{d} \leqslant 5$.

From there we can easily deduce the following corollary.

Corollary 3. Suppose $G$ has maximum degree 5. Then $\chi_{d}(G) \leqslant 7$.

Analyzing a greedy colouring approach, Haxell, Wilfong and Winkler showed that in general $\Delta+o(\Delta)$ colours suffice.

Theorem 4 (Haxell, Wilfong, Winkler [6]). For each bipartite graph $G, \chi_{d}(G) \leqslant \Delta(G)+$ $c \Delta(G)^{\frac{1}{2}}$ for some universal constant $c$.

Alon and Asodi [1] later improved the bound for simple bipartite graphs to $\Delta+o$ (1) $\Delta$. In the same paper they proved Haxell et al.'s conjecture for even multicycles in the case where $\Delta+1$ is prime. In the next section as a brief historical aside, we sketch the real-world motivation for this problem as described by Saniee and Wadjaja.

\section{Motivation}

The delay-colouring of bipartite graphs came about from the scheduler required in an alternative approach to optical transport networks which we briefly outline here. Introduced by Saniee and Widjaja [9], Time-domain Wavelength Interleaved Networking (TWIN) sought to overcome the bandwidth inefficiency of traditional optical circuit switching (due to most end-to-end applications only requiring a small fraction of the wavelength capacity) and avoid the overhead of optical-to-electronic conversion of data. The technologies 


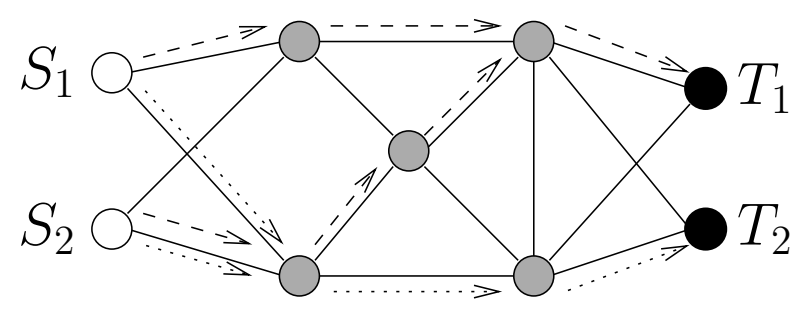

Figure 2: An example TWIN architecture. The white and black nodes, labelled $S_{i}$ and $T_{i}$, are the source and destination edge-nodes, respectively; all remaining nodes are core. The route in-arborescences for $T_{1}$ and $T_{2}$ are indicated with dashed and dotted arrows.

underpinning these networks are ultra-fast tunable lasers that can switch wavelengths in nanoseconds and wavelength-selective cross-connects that route the optical signals, or bursts, based solely on their wavelength.

Figure 2 illustrates an example of the TWIN architecture. The network is made up of two types of nodes. The edge-nodes, responsible for sending and receiving data, are each assigned a unique set of wavelengths which can be thought of as their address. Corenodes of the network use this address information to forward packets of data on to their destination. The routes that the data packets follow inside the network are predetermined as in-arborescences, ${ }^{1}$ one for each destination, whose leaves are the edge nodes and whose root is the destination.

Each source edge-node is equipped with an ultra-fast tunable laser that sends bursts to a specific destination by tuning the laser to the corresponding wavelength. Since the laser can only be at one state at any point of time, each edge-node must schedule its transmissions such that each time slot corresponds to at most one destination wavelength. Core-nodes receive these bursts of data and passively forward them on along the predetermined routes using wavelength-selective cross-connects that can simultaneously forward packets, provided they arrive on different wavelengths. Since the routes are arborescences, it is easy to see that if bursts of data destined for a common destination edge-node do not overlap at that destination then they do not overlap at any core-node. Hence, in scheduling the bursts it is enough to ensure that the bursts scheduled to arrive at each destination arrive at unique time slots.

This scheduling can be modelled as the edge-colouring of a bipartite graph $G=$ $(X, Y, E)$, where the nodes of $X$ correspond to the source edge-nodes, $Y$ the destination edge-nodes, and there exists an edge $x y \in E, x \in X$ and $y \in Y$, if $x$ will send data to $y$. If transmissions are instantaneous then this is exactly the well-understood bipartite edge-colouring for which $\Delta(G)$, the maximum degree of $G$, colours (or timeslots) suffice [7]. In reality, transmissions are not instantaneous and so we must produce a scheduling where the delay of transmissions affects the arrival time and hence must be accommodated in the model. This is exactly the $\tau$-delay-colouring problem described

\footnotetext{
${ }^{1}$ An in-arborescence is a directed graph $H$ with root $r$ satisfying for each vertex $u \in H$ there is exactly one directed path from $u$ to $r$.
} 
above.

It is worth noting that much work has been focused on heuristics for determining feasible schedules. Since our focus is on proving Conjecture 1, we refer the interested reader to $[9,8]$ for further information.

\section{Preliminaries}

In order to prove Theorem 2, it is enough to show it holds for 4-regular graphs, since every bipartite graph $G$ is a subgraph of a $\Delta(G)$-regular bipartite graph. In what follows, $G$ is a 4-regular bipartite graph. Its vertices $V(G)$ are partitioned into sets $(X, Y)$ and we assume $|X|=|Y|=n$ and $|E(G)|=m=4 n$. We are also given a delay function $r: E(G) \rightarrow \mathbb{N}$.

We will need to extend the notion of delay colouring to its list version. Let $G$ be a bipartite graph and $F$ be a field. Suppose $\mathcal{S}=\left\{\mathcal{S}_{e} \subseteq F: e \in E(G)\right\}$ is a collection of lists. We say that $G$ is $\mathcal{S}$-list-delay-colourable (in $F$ ) if for every delay function $r$, the instance $(G, r)$ has a delay colouring $f_{e}$ where $f_{e} \in \mathcal{S}_{e}$ for each edge $e$. We say $G$ is $k$-list-delay-colourable in $F$ is $G$ if $\mathcal{S}$-list-delay-colourable for every choice of $\mathcal{S}$ satisfying $\forall S_{e} \in \mathcal{S},\left|S_{e}\right| \geqslant k$. When $F=\mathbb{Z}_{\tau}$ then we simply say $G$ is $k$-list-delay-colourable $(\bmod \tau)$.

Before getting to the proof, let us recall some pertinent definitions. In the proof, we will need to work both in the graph $G$ and in its line graph $L(G)$. We remind the reader that $L(G)$ is the graph with vertex set $E(G)$ where two vertices are adjacent if and only if their corresponding edges meet in $G$.

An orientation of a graph $G$ is a directed graph obtained from $G$ by assigning a direction to each edge. If $e=(u, v)$ is an edge in a directed graph, we say that $u$ is the tail of $e$ and $v$ is the head. Also, for each vertex $v$ in a directed graph, the outdegree $d^{+}(v)$ denotes the number of edges with tail $v$ and the indegree $d^{-}(v)$ the number of edges with head $v$. A tournament is an oriented complete graph, and a transitive tournament is a tournament whose vertices can be ordered in such a way that the tail of each edge is smaller (with respect to this ordering) than its head. Observe that in an $n$-vertex transitive tournament, the sequence of outdegrees equals $(n-1, n-2, \ldots, 0)$.

Suppose $G=G_{1} \oplus G_{2} \oplus \cdots \oplus G_{n}$, where each $G_{i}$ is a complete graph (the symbol $\oplus$ denotes disjoint union with respect to edges). Defined in [4], a clique-transitive orientation of $G$ is an orientation of $G$ with the property that on each of the particular cliques $G_{i}$ of $G$ the induced orientation is a transitive tournament. This implies that the outdegrees given by a clique transitive orientation of $G$ within a certain $G_{i}$ (i.e. the number of outgoing edges from a vertex in $G_{i}$ to other vertices in $G_{i}$ ) are exactly the numbers $k-$ $1, k-2 \ldots, 0$, where $k$ is the size of clique $G_{i}$. Note that the term clique-transitive always refers to a particular clique decomposition of $G$, and other cliques are not necessarily transitively oriented. Observe also that a transitive tournament is completely defined by its outdegrees, and hence a clique transitive orientation of a graph that is the edge disjoint union of cliques is completely defined by the out-degrees within the cliques. 


\section{Tools}

We will need Alon and Tarsi's Combinatorial Nullstellensatz theorem, proved in [2].

Theorem 5 (Alon,Tarsi [2]). Let $F$ be an arbitrary field, and let $P=P\left(x_{1}, \ldots, x_{n}\right)$ be a polynomial in $F\left[x_{1}, \ldots, x_{n}\right]$. Suppose that the degree of $P$ is $\sum_{i=1}^{n} t_{i}$, where each $t_{i}$ is a non-negative integer, and suppose the coefficient of $\prod_{i=1}^{n} x_{i}^{t_{i}}$ in $P$ is nonzero. Then, if $S_{1}, \ldots, S_{n}$ are subsets of $F$ with $\left|S_{i}\right|>t_{i}$, there are $s_{1} \in S_{1}, s_{2} \in S_{2}, \ldots, s_{n} \in S_{n}$ so that $P\left(s_{1}, \ldots, s_{n}\right) \neq 0$.

In [2], Alon and Tarsi gave the following application of Theorem 5 to list colouring. Let $G$ be an undirected graph on an ordered vertex set $V=v_{1}, \ldots, v_{n}$. Suppose $\rho: V(G) \rightarrow \mathbb{N}$ is a map from the vertices to natural numbers. An orientation of the edges of $G$ is said to obey $\rho$ if we have $d^{+}(v)=\rho(v)$ for each $v \in V(G)$. Given an orientation of the edges of $G$, a directed edge $\left(v_{i}, v_{j}\right)$ is said to be reversed if $i>j$. The parity of an orientation is the parity of its number of reversed edges. Fix $\rho$, then $D E_{G}(\rho)$ and $D O_{G}(\rho)$ denote the number of even and odd orientations of $G$ obeying $\rho$, respectively.

Theorem 6 (Alon,Tarsi [2]). Let $G$ be a graph on an ordered vertex set $V$. Let $\mathcal{S}=\left(S_{v}\right.$ : $v \in V)$ be a collection of subsets of $F$ and $\rho: V \rightarrow \mathbb{N}^{+}$such that $\rho(v)<\left|S_{v}\right|$ for each $v$. If $D E_{G}(\rho) \neq D O_{G}(\rho)$ then $G$ has an $\mathcal{S}$-list colouring.

We can adapt the proof of Theorem 6 to get the result for (list) delay edge colouring as follows.

Lemma 7. Let $(G, r)$ be an instance of delay colouring. Assume that $G$ is $\Delta$-regular, and denote its edges $e_{1}, \ldots, e_{m}$ (via an arbitrary ordering). Let $\rho: E(G) \rightarrow \mathbb{N}$ be a map from the edges of $G$ (i.e. vertices of $L(G)$ ) to the natural numbers and $\tau \in \mathbb{N}^{+}$ be a prime. Suppose $D E_{L(G)}(\rho) \neq D O_{L(G)}(\rho)(\bmod \tau)$. Then for any choice of lists $\mathcal{S}=\left(S_{1}, \ldots, S_{m}\right) \subseteq \mathbb{Z}_{\tau}$ with $\left|S_{i}\right|>\rho\left(e_{i}\right), G$ has an $\mathcal{S}$-list delay colouring.

Proof. Consider the polynomial with coefficients in the field $\mathbb{Z}_{\tau}$ defined as follows. Let

$$
P\left(x_{1}, \ldots, x_{m}\right)=\prod_{\substack{e_{i} e_{j} \in E(G) \\ e_{i} \cap e_{j} \in X \\ i<j}}\left(x_{i}-x_{j}\right) \prod_{\substack{e_{i} e_{j} \in E(G) \\ e_{i} \cap e_{j} \in Y \\ i<j}}\left(x_{i}+d_{i}-x_{j}-d_{j}\right) .
$$

It is easy to see that a solution to $P\left(x_{1}, \ldots, x_{m}\right) \neq 0$ with $x_{i} \in \mathbb{Z}_{\tau}$ corresponds to a valid delay colouring of $G$.

We interpret the monomials of maximum degree in $P$ in terms of orientations of $L(G)$. Each such monomial in fact has the same degree and has the form $x_{1}^{t_{1}} \ldots x_{m}^{t_{m}}$, where $t_{1}+\cdots+t_{m}=|E(L(G))|=n \Delta(\Delta-1)$. Choose one such monomial and set $\rho\left(e_{i}\right)=t_{i}$ for each $i$; we claim that the coefficient of $x_{1}^{t_{1}} \ldots x_{m}^{t_{m}}$ in the expansion of $P$ equals $D E_{L(G)}(\rho)-D O_{L(G)}(\rho)$. To see this, multiply out the terms of $P$ and observe that there is a one-to-one correspondence between the monomials of maximum degree in the expansion and multisets of vertices obtained by choosing one vertex from each edge of $L(G)$. In other 
words, each one corresponds to an orientation of $L(G)$ (choose the vertex corresponding to the tail of each edge in the orientation). Moreover, a term appears with coefficient -1 if the number of reversed edges in the corresponding orientation is odd, and with coefficient 1 if the number of reversed edges is even. Thus the coefficient of the term $x_{1}^{\rho\left(e_{1}\right)} \ldots x_{m}^{\rho\left(e_{m}\right)}$ is exactly $D E_{L(G)}(\rho)-D O_{L(G)}(\rho)$. By assumption $D E_{L(G)}(\rho) \neq D O_{L(G)}(\rho)(\bmod \tau)$, and so, the result follows immediately from Theorem 5 .

To apply Lemma 7, we need to count orientations of $L(G)$. Since $L(G)$ is a line graph, there is a natural partition of its edges into $\Delta$-cliques, each one corresponding to a vertex of $G$, such that each vertex of $L(G)$ belongs to exactly two of the cliques. Let $L(G)=C_{1} \oplus \cdots \oplus C_{2 n}$ denote this decomposition of $E(G)$ into cliques.

In [4], Häggkvist and Janssen applied Lemma 6 to determine the list chromatic index of the complete graph. There, they found that when counting orientations, it is sufficient to restrict ones attention to clique-transitive orientations. We will also apply this simplification.

Lemma 8 (Häggkvist,Janssen [4]). If $G=G_{1} \oplus G_{2} \oplus \cdots \oplus G_{n}$ where each $G_{i}$ is a complete graph, then for each map $\rho: V(G) \rightarrow \mathbb{N}$, the number of even orientations obeying $\rho$ which are not clique-transitive is equal to the number of odd such orientations.

Let $D E^{\prime}(\rho)$ and $D O^{\prime}(\rho)$ respectively count the number of even and odd clique-transitive orientations of $L(G)$ obeying $\rho$. Lemma 8 says that it is enough to have $D E_{L(G)}^{\prime}(\rho) \neq$ $D O_{L(G)}^{\prime}(\rho)(\bmod \tau)$ in the hypothesis of Lemma 7 .

\section{Proof of the main result}

We can now prove Theorem 2 .

Lemma 9. Suppose $G$ is 4-regular. Then $G$ is 5-delay-colourable.

Proof. We work in the line graph $L(G)$. By a classical theorem of König [7], the graph $L(G)$ has a vertex 4-colouring. Fix such a colouring, and denote the colours by $\alpha, \beta, \delta, \gamma$. Define the map $\rho: V(L(G)) \rightarrow\{2,4\}$ as follows.

$$
\rho(v)= \begin{cases}4, & \text { if } v \text { has colour } \in\{\alpha, \beta\} \\ 2, & \text { if } v \text { has colour } \in\{\delta, \gamma\} .\end{cases}
$$

We will show that the number of $\rho$-obeying clique-transitive orientations is a power of 2 , and that all $\rho$-obeying clique-transitive orientations have the same parity. This suffices to show that $D E^{\prime}(\rho) \neq D O^{\prime}(\rho)(\bmod 5)$, and we can apply Lemma 7 with the field $\mathbb{Z}_{5}$ to complete the proof.

Recall that the edges of $L(G)$ can be partitioned into 4-cliques $C_{1}, \ldots, C_{2 n}$, such that each vertex belongs to exactly two cliques. Consider a single clique $C_{i}$. For any cliquetransitive orientation, the four vertices in $C_{i}$ have outdegrees $3,2,1,0$ within $C_{i}$. We claim that for any $\rho$-obeying clique-transitive orientation, the vertices with outdegrees 3 and 1 
have colours in $\{\alpha, \beta\}$ (and thus $\rho=4$ ) and the vertices with outdegrees 2 and 0 have colours in $\{\gamma, \delta\}$ (and thus $\rho=2$ ). To see this, note that a vertex with $\rho=2$ clearly cannot have outdegree 3 in $C_{i}$. Also, a vertex with $\rho=4$ cannot have outdegree 0 in $C_{i}$, because it can have outdegree at most 3 in the other clique it belongs to. Given that the vertices with outdegrees 3,0 respectively have $\rho=4,2$ respectively, there are two possibilities: either the vertices of outdegree $3,2,1,0$ have $\rho=4,2,4,2$ respectively, or they have $\rho=4,4,2,2$ respectively. In the first possibility, the vertices respectively have outdegrees $1,0,3,2$ in their other cliques, and in the second the vertices have outdegrees 1,2,1,2 in their other cliques. But in total, for each $i \in\{0,1,2,3\}$, there are $2 n$ vertices which have outdegree $i$ in some clique, and we deduce by counting that the second possibility cannot happen.

Thus for any $\rho$-obeying clique-transitive orientation, each clique has one of 4 colouringtypes, depending on the sequence of colours in the transitive ordering- that is $(\alpha, \delta, \beta, \gamma)$ or $(\alpha, \gamma, \beta, \delta)$ or $(\beta, \delta, \alpha, \gamma)$ or $(\beta, \gamma, \alpha, \delta)$ (in decreasing order of degree). Each colouring-type is uniquely identified by the colours of the first two vertices, that is $(\alpha, \delta),(\alpha, \gamma),(\beta, \delta)$ or $(\beta, \gamma)$.

We first note that such a $\rho$-obeying clique-transitive orientation exists. It is enough to show that we can assign every vertex in each clique $C_{i}$ a unique outdegree from $\{0,1,2,3\}$ such that if $v \in C_{i} \cap C_{j}$ then the outdegree of $v$ in $C_{i}$ plus its outdegree in $C_{j}$ equals $\rho(v)$. Let $G_{\alpha \beta}$ denote the subgraph of $L(G)$ induced on the vertices coloured $\alpha$ or $\beta$, equivalently, those vertices with $\rho=4$. Each connected component of $G_{\alpha \beta}$ is an even cycle containing exactly two vertices from each $C_{i}$, denote these components $H_{\alpha \beta}^{1}, \ldots, H_{\alpha \beta}^{r}$. Each vertex of $H_{\alpha \beta}^{j}$ is necessary assigned outdegrees 1,3 in the two cliques containing it. Clearly fixing the outdegrees of one such vertex forces the remaining outdegrees of the other vertices in $H_{\alpha \beta}^{j}$. We can similarly assign outdegrees of 0,2 to the vertices with $\rho=2$ by considering $G_{\delta \gamma}$ and $H_{\delta \gamma}^{1}, \ldots, H_{\delta \gamma}^{s}$. Since each vertex is in exactly one of $G_{\alpha \beta}$ and $G_{\delta \gamma}$, it follows that this is the desired assignment of outdegrees.

Let $\mathcal{C}\left(H_{\alpha \beta}^{i}\right)=\left\{C_{j}: C_{j} \cap H_{\alpha \beta}^{i} \neq \emptyset\right\}$, and note that the sets $\mathcal{C}\left(H_{\alpha \beta}^{i}\right)$ partition the cliques $C_{1}, \ldots, C_{2 n}$. Similarly, define the partition $\mathcal{C}\left(H_{\delta \gamma}^{i}\right)$.

In each component $H_{\alpha \beta}^{i}$, every pair of adjacent vertices belongs to a common clique $C_{j} \in \mathcal{C}\left(H_{\alpha \beta}^{i}\right)$. Thus, the first colour in the colouring-type of $C_{j}$ uniquely determines the first colour in the colouring-type of every clique in $\mathcal{C}\left(H_{\alpha \beta}^{i}\right)$ (they alternate along the cycle). Similarly, the second colour of the colouring-type of any clique $C_{j} \in \mathcal{C}\left(H_{\delta \gamma}^{i}\right)$ uniquely determines the colour of the second vertex in every clique in $\mathcal{C}\left(H_{\delta \gamma}^{i}\right)$. In other words, the colouring-type of each clique $C_{j}$ determines the colouring-type of each clique in $\mathcal{C}\left(H_{\alpha \beta}^{i}\right) \cup \mathcal{C}\left(H_{\delta \gamma}^{k}\right)$, where $H_{\alpha \beta}^{i}$ and $H_{\delta \gamma}^{k}$ are the two components meeting $C_{j}$. We deduce that there are $2^{r+s}$ clique-transitive orientations of $L(G)$ which obey $\rho$.

It remains to show that any two such orientations have the same parity. The above discussion implies that any $\rho$-obeying orientation can be obtained from another by performing a sequence of operations, each one consisting of either

- changing the first colour of the colouring-type of all cliques in $\mathcal{C}\left(H_{\alpha \beta}^{i}\right)$ (either from $\alpha$ to $\beta$ or from $\beta$ to $\alpha$ ) for some $i$, or

- changing the second colour of the colouring-type of all cliques $\mathcal{C}\left(H_{\delta \gamma}^{i}\right)$ (either from 
$\delta$ to $\gamma$ or from $\gamma$ to $\delta$ ) for some $i$.

It's easy to see that one such operation preserves the parity of the orientation, since in the process the direction of an even number of edges is changed. To see this, consider that $\left|\mathcal{C}\left(H_{\alpha \beta}^{i}\right)\right|$ is even and we change the direction of three edges per clique. It follows that performing a sequence of such operations leaves the parity unchanged.

Note that the proof of Lemma 9 in fact shows something a bit stronger: for each prime $p \geqslant 5$ that $G$ is 4 -list-delay-colourable $(\bmod p)$. Finally, we deduce Corollary 3.

Proof of Corollary 3. Let $(G, r)$ be an instance of delay colouring with $\Delta(G)=5$. Let $M$ be a perfect matching in $G$. From Lemma 9, the graph $G \backslash M$ is 5 -list-delay-colourable. For each edge $e \notin M$, let $e^{\prime}$ denote the unique edge in $M$ that meets $e$ in $Y$. Define $\mathcal{S}_{e}=\mathbb{Z}_{7} \backslash\left\{0, r_{e^{\prime}}\right\}$. Let $f_{e}$ be a delay colouring of $G \backslash M$ from the lists $S_{e}$. Then, for each edge $e \in M$ set $f_{e}=0$. The colouring $f_{e}$ is a 7-delay-colouring, proving the corollary.

\section{References}

[1] Noga Alon and Vera Asodi. Edge coloring with delays. In Approximation, Randomization, and Combinatorial Optimization. Algorithms and Techniques, pages 237-248. Springer, 2004.

[2] Noga Alon and Michael Tarsi. Colorings and orientations of graphs. Combinatorica, 12(2):125-134, 1992.

[3] Agelos Georgakopoulos. Delay colourings of cubic graphs. The Electronic Journal of Combinatorics, 20(3):P45, 2013.

[4] Roland Häggkvist and Jeannette Janssen. New bounds on the list-chromatic index of the complete graph and other simple graphs. Combinatorics, Probability and Computing, 6(3):295-313, 1997.

[5] Marshall Hall. A combinatorial problem on abelian groups. Proceedings of the American Mathematical Society, 3(4):584-587, 1952.

[6] Penny Haxell, Gordon Wilfong, and Peter Winkler. Delay coloring and optical networks. preprint, 2001.

[7] Dénes König. Graphok és alkalmazásuk a determinánsok és a halmazok elméletére. Mathematikai és Természettudományi Ertesito, 34:104-119, 1916.

[8] Iraj Saniee and Indra Widjaja. Design and performance of randomized schedules for time-domain wavelength interleaved networks. Bell Labs Technical Journal, 14(2):97111, Summer 2009.

[9] Indra Widjaja and Iraj Saniee. Simplified layering and flexible bandwidth with TWIN. In Proceedings of the ACM SIGCOMM Workshop on Future Directions in Network Architecture, FDNA '04, pages 13-20, New York, NY, USA, 2004. ACM. 